Supplementary Informationfor

\title{
Effect of Strong Intermolecular Interaction in 2D Inorganic Molecular Crystals
}

Xin Feng, ${ }^{+, \perp}$ Xingliang Peng, ${ }^{+, \perp}$ Baixin Peng, $₫$ Zexin Li,, Wentao Huang, ${ }^{+}$Sijie Yang, ${ }^{\dagger}$ Ke Pei, † Zongdong Sun, † Fuqiang Huang, §,॥ Huiqiao Li, † Zhigang Shuai, ,, † Tianyou Zhai $*,+$

$\dagger$ State Key Laboratory of Material Processing and Die \& Mould Technology, and School of Materials Science and Engineering, Huazhong University of Science and Technology, Wuhan 430074, P. R. China

‡ MOE Key Laboratory of Organic OptoElectronics and Molecular Engineering, Department of Chemistry, Tsinghua University, Beijing 100084, P. R. China

$\S$ State Key Laboratory of High Performance Ceramics and Superfine Microstructure, Shanghai Institute of Ceramics, Chinese Academy of Sciences, Shanghai200050, P. R. China

" State Key Laboratory of Rare Earth Materials Chemistry and Applications, College of Chemistry and Molecular Engineering, Peking University, Beijing 100871, P. R. China 


\section{List of contents:}

1. Materials and methods

1) Synthesis of $\alpha-\mathrm{P}_{4} \mathrm{Se}_{3}$ Powders and $2 \mathrm{D} \mathrm{P}_{4} \mathrm{Se}_{3}$ Nanoflakes

2) Characterization

3) Theoretical calculation

2. Supplementary figures and tables 


\section{Materials and methods}

\section{1) Synthesis of $\alpha-P_{4} \mathrm{Se}_{3}$ Powders and $2 \mathrm{D} \mathrm{P}_{4} \mathrm{Se}_{3}$ Nanoflakes}

$\alpha-\mathrm{P}_{4} \mathrm{Se}_{3}$ powders were synthesized with solid-state reaction. The red $\mathrm{P}$ (Alfa 99.9\%) and black Se powders (Aladdin 99.9\%) were mixed in molar ratio, ground, and then sealed in a quartz tube with a vacuum condition about $10^{-5}$ Torr. The tube was slowly heated up to $400{ }^{\circ} \mathrm{C}$, kept at this temperature for $48 \mathrm{~h}$, and then cooled to room temperature within $48 \mathrm{~h}$. The obtained product was further purified by recrystallization from excess carbon disulfide under an atmosphere of argon. 2D $\mathrm{P}_{4} \mathrm{Se}_{3}$ nanoflakes were prepared by a facile drop-casting process. Typically, $20 \mathrm{mg}$ of $\alpha$ $\mathrm{P}_{4} \mathrm{Se}_{3}$ powder was added in $2 \mathrm{ml}$ of carbon disulfide to obtain a supersaturated solution. After being stirred vigorously, a drop of this supersaturated solution about $50 \mu \mathrm{l}$ was dripped on the glass substrate inside a covered petri dish under argon atmosphere, allowing the solvent to volatilize slowly. Finally, 2D $\mathrm{P}_{4} \mathrm{Se}_{3}$ nanoflakes can be found on the glass substrate.

\section{2) Characterization}

The as-synthesized $\alpha-\mathrm{P}_{4} \mathrm{Se}_{3}$ and $2 \mathrm{D} \mathrm{P}_{4} \mathrm{Se}_{3}$ nanoflakes were characterized by XRD (D2 Phaser, Bruker) and Raman spectroscopy (Alpha 300RS+, WITEC). The morphology, thickness and element information of $2 \mathrm{D} \mathrm{P}_{4} \mathrm{Se}_{3}$ nanoflakes were characterized with the optical microscope (BX53M, OLYMPUS), atomic force microscope (Dimension Icon, Bruker), scanning electron microscope (Quanta 650 FEG, FEI), and X-ray photoelectron spectrometer (AXIS-ULTRA DLD-600W, Kratos). Single-crystal X-ray diffraction was performed at room temperature by a Bruker D8QUEST diffractometer equipped with Mo $\mathrm{K} \alpha$ radiation and the crystal structure was solved and refined using APEX3 program. ${ }^{1}$ Absorption corrections were performed using the multiscan method (ASDABS). Absorption and in-situ temperature-dependent absorption spectra were collected with a Jasco MSV-5200 microscopic spectrophotometer. The electrical tests were perfromed on the semiconductor characterization system (B1500A, Agilent) combined with a probe station (TTPX, LakeShore). 


\section{3) Theoretical calculation}

The geometry of single molecule is optimized at B3LYP/6-311+G** level, and the HOMO and LUMO orbitals are calculated at the same level based on optimized geometry. The total charge density distribution is calculated by employing periodic boundary conditions using the Vienna ab initio simulation package (VASP) ${ }^{2}$. The exchange correlation functional was described by Perdew-Burke-Ernzerhof (PBE) functional combining with the generalized gradient approximation (GGA) ${ }^{3}$, and the kinetic energy cutoff is set above $400 \mathrm{eV}$. The inter-molecular transfer integral is calculated at B3LYP/6-311+G** level based on single crystal stacking. The vibrational modes analysis for reorganization energy calculations are based on the optimized configurations obtained by quantum mechanical and molecular mechanical calculations $(\mathrm{QM} / \mathrm{MM})$, where the central one molecule (free) is treated by QM (B3LYP/6-311+G**) and the surrounding molecules (fixed) are treated by MM (UFF). The surrounding molecules are those whose center of mass (COM) to the COM of central molecule is less than $20 \AA$. The hop rates between the adjacent molecules are calculated by Marcus theory ${ }^{4}$ and quantum nuclear tunneling model ${ }^{5-9}$. The charge carrier mobilities are obtained by performing 2000 kinetic Monte Carlo (KMC) simulations based on the hop rates. The calculations of transfer integral, reorganization energy, hop rate and mobility are performed by MOMAP 2021A software ${ }^{10}$, and the structural optimization and single point calculations are performed by GAUSSIAN 16 A03 program ${ }^{11}$. It should be noted that Marcus theory or equivalently the quantum nuclear tunneling model is applicable under the assumption of charge localization. If the latter arises from intramolecular relaxation lambda, then, lambda should be much larger than intermolecular interaction V. This is not always the case. We also note that for organic materials, especially for low-dimensional systems, there exist a number of mechanisms for charge localization, including static and dynamic disorders, and various impurities. Especially, we note that the transient localization theory ${ }^{12}$ has emerged quickly in recent years. These are quite complicated and challenging for theoretical description and are far beyond the scope of the present work. 
2. Supplementary figures and tables

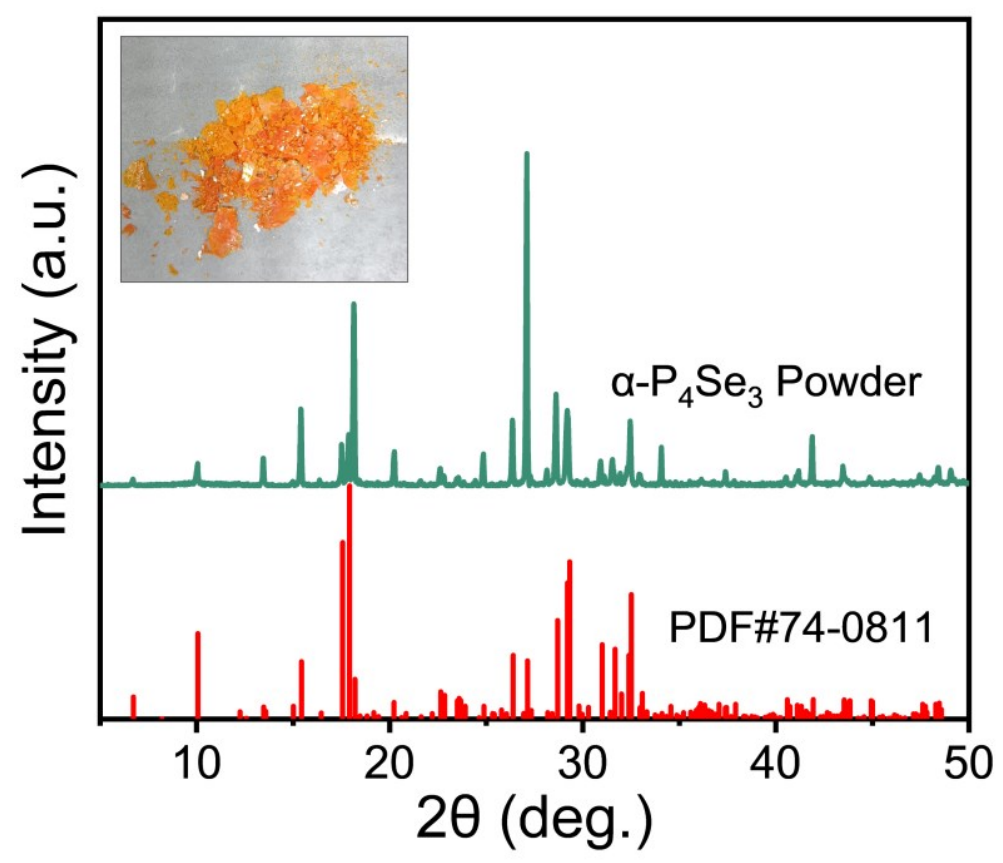

Figure S1. XRD pattern of as-synthesized $\alpha-\mathrm{P}_{4} \mathrm{Se}_{3}$ powders. 

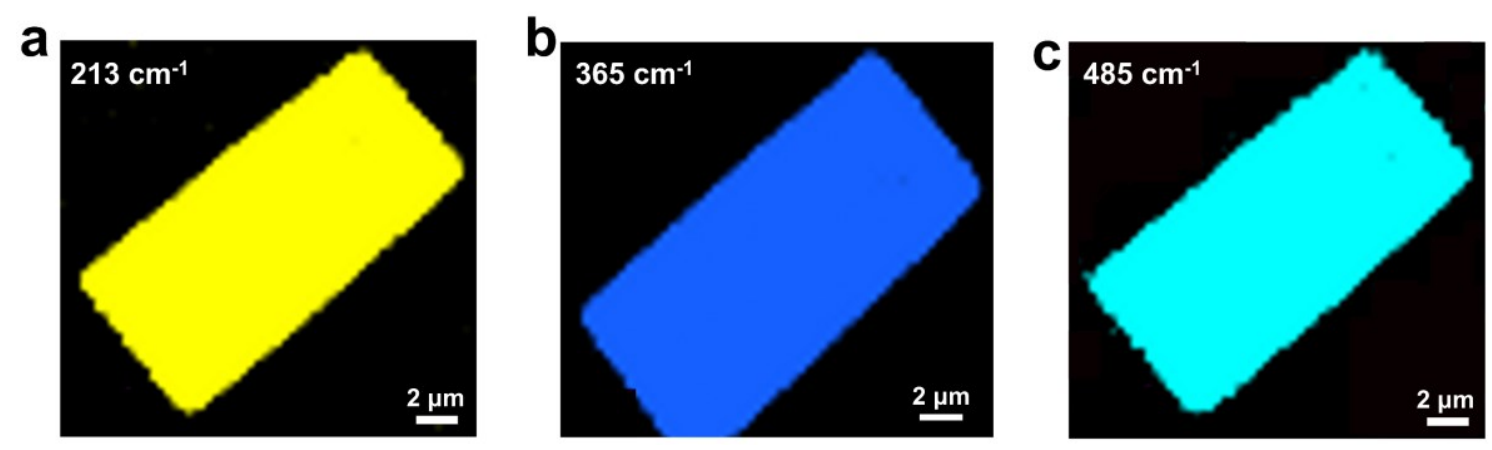

Figure S2. (a, b c) Spatially resolved Raman mapping of three typical internal modes at $213 \mathrm{~cm}^{-1}, 365 \mathrm{~cm}^{-1}$ and $485 \mathrm{~cm}^{-1}$, respectively. 


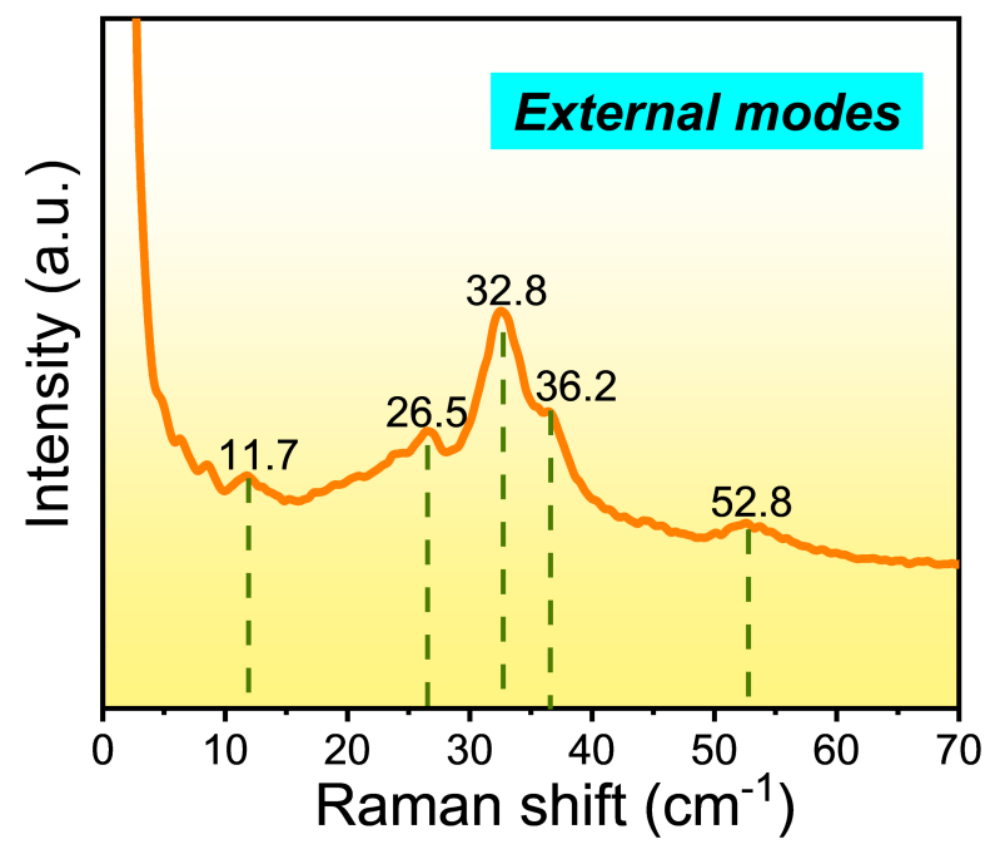

Figure S3. Low wavenumber Raman modes of the as-synthesized $2 \mathrm{D} \mathrm{P}_{4} \mathrm{Se}_{3}$ nanoflakes. 

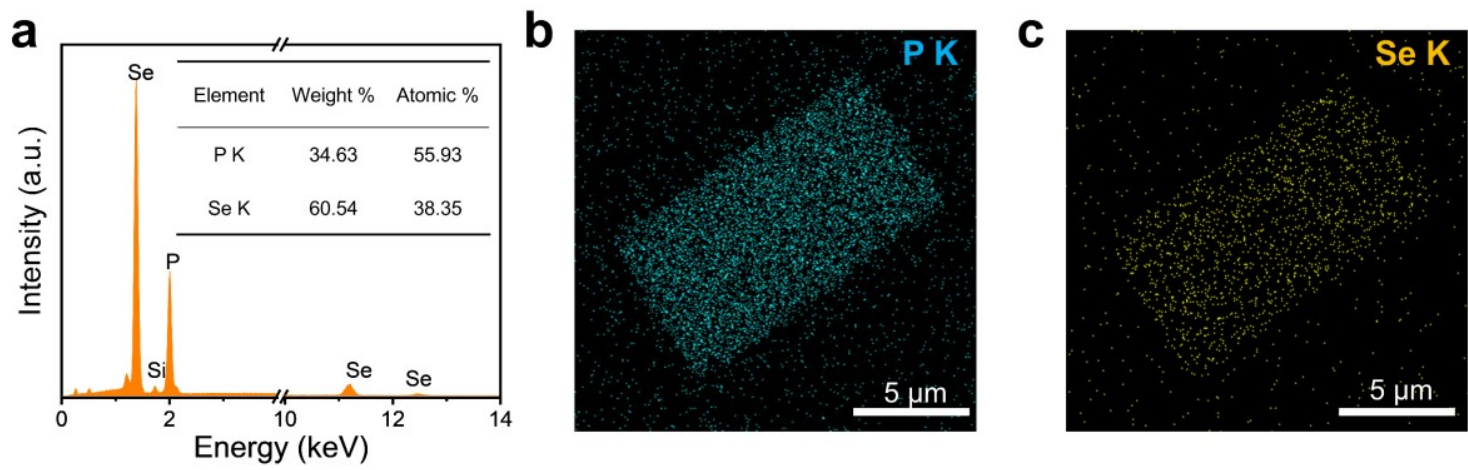

Figure S4. (a) EDS spectrum of as-synthesized 2D $\mathrm{P}_{4} \mathrm{Se}_{3}$ nanoflake. (b, c) Elemental mapping of $\mathrm{P}$ and Se elements, respectively. 

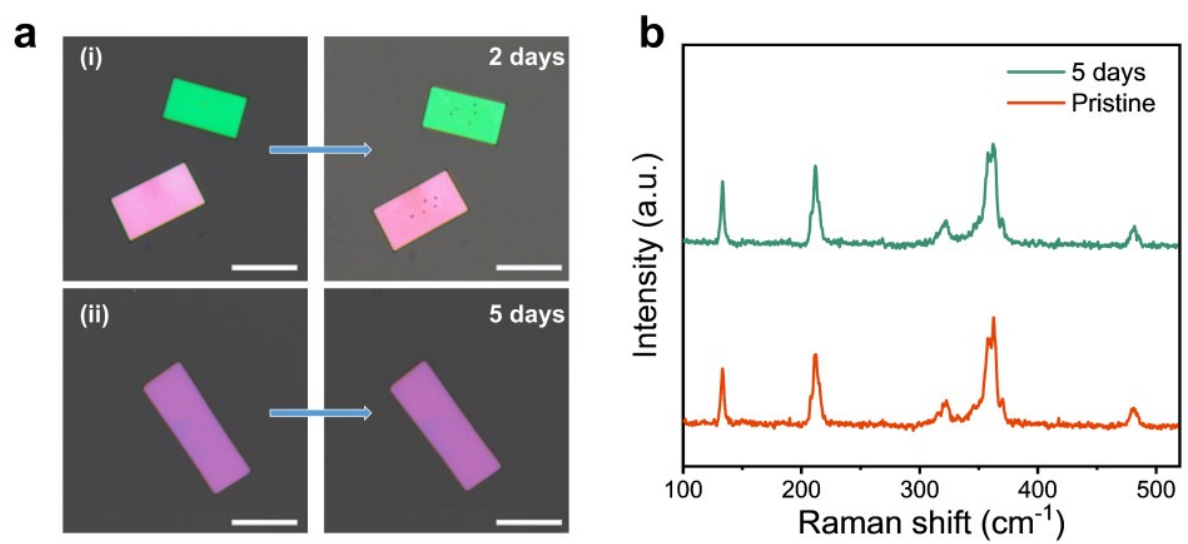

Figure S5. (a) Optical images for the two group of $\alpha-\mathrm{P}_{4} \mathrm{Se}_{3}$ nanoflake placed in an open environment (i) and the environment without water and oxygen (ii). Scale bar, $10 \mu \mathrm{m}$. (b) Raman spectra for the $\alpha-\mathrm{P}_{4} \mathrm{Se}_{3}$ nanoflake placed in the environment without water and oxygen, group (ii). 


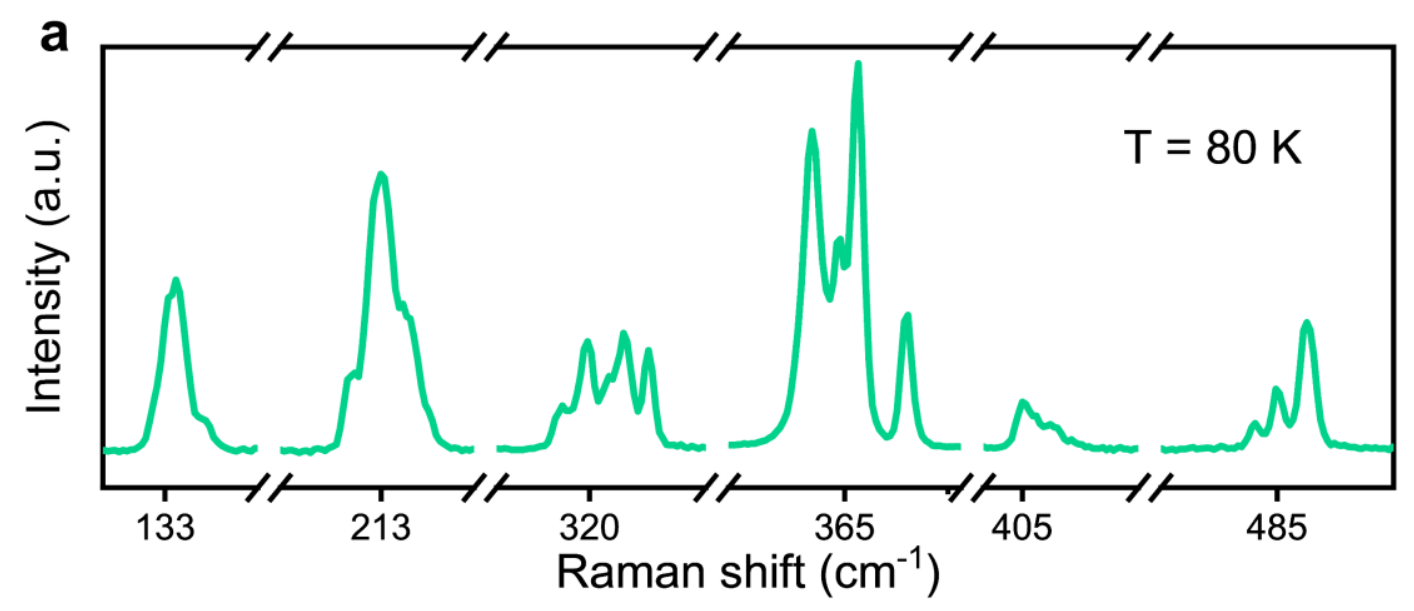

b
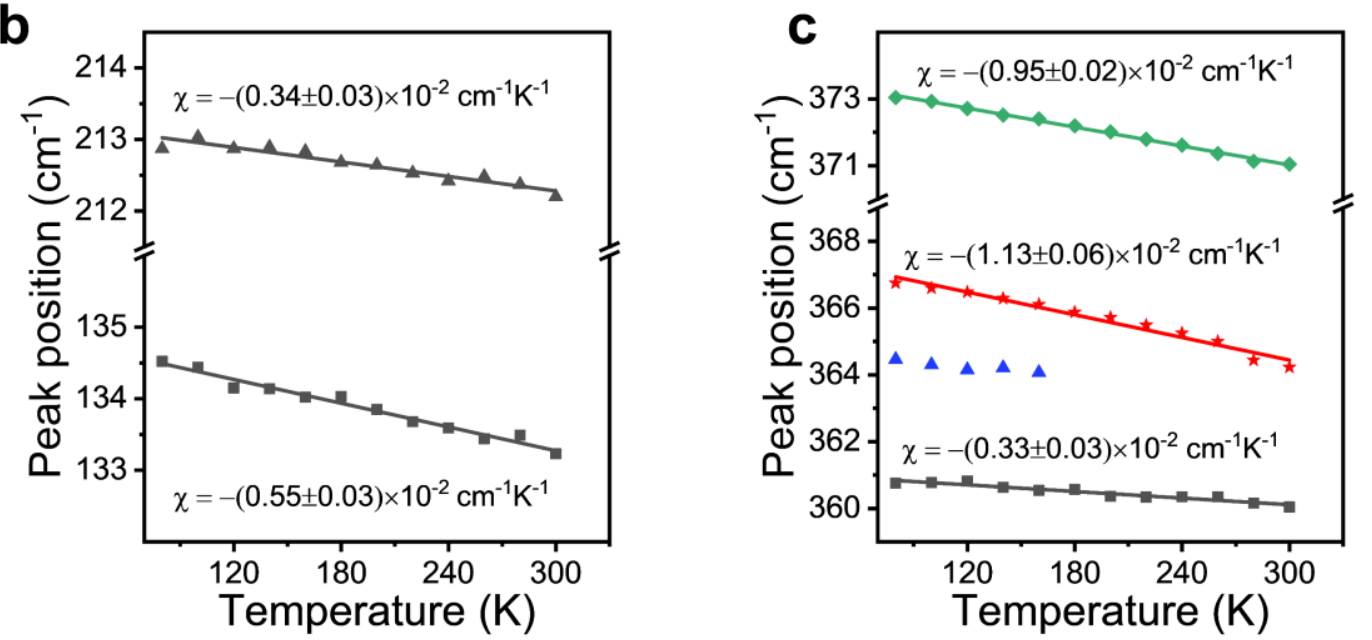

Figure S6. (a) Raman spectrum of a $\mathrm{P}_{4} \mathrm{Se}_{3}$ nanoflake at $80 \mathrm{~K}$. (b, c) Temperature dependence of Raman peaks at $134.5 \mathrm{~cm}^{-1}, 213 \mathrm{~cm}^{-1}, 361 \mathrm{~cm}^{-1}, 367 \mathrm{~cm}^{-1}$ and $373 \mathrm{~cm}^{-1}$, and the corresponding first order temperature coefficients $(\chi)$. 

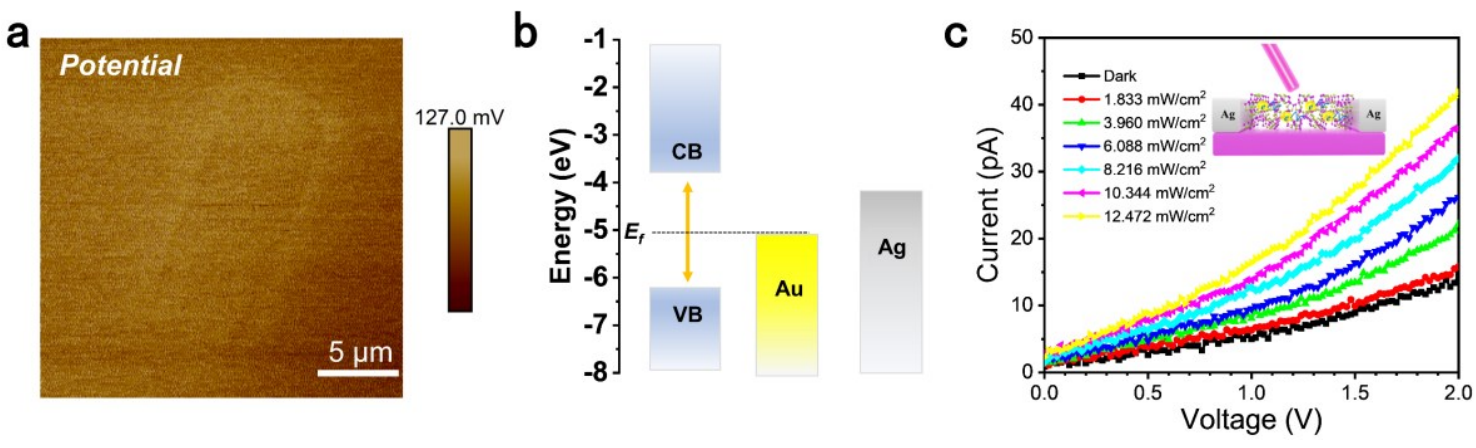

Figure S7. (a) KPFM image of the $\mathrm{P}_{4} \mathrm{Se}_{3}$ nanoflake on the gold substrate. (b) Band structure of the $\mathrm{P}_{4} \mathrm{Se}_{3}$ nanoflake. (c) $I-V$ curves under dark and $365 \mathrm{~nm}$ laser illumination with different power intensities. 


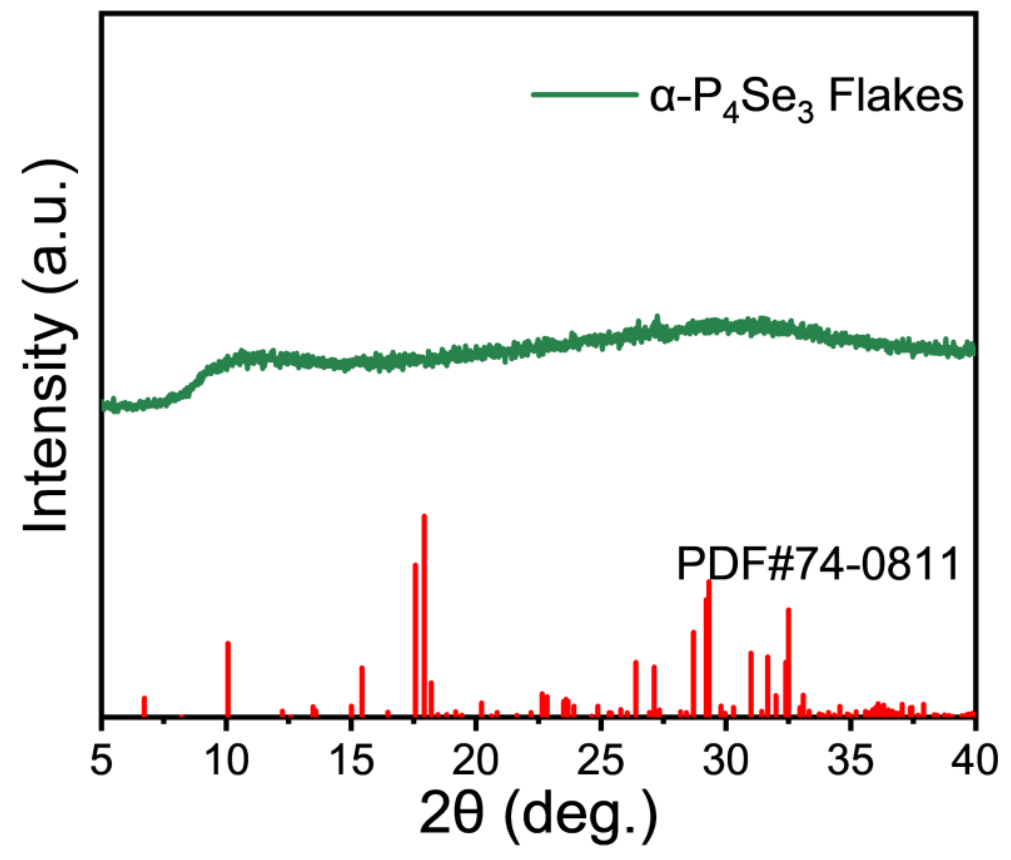

Figure S8. XRD pattern of $\alpha-\mathrm{P}_{4} \mathrm{Se}_{3}$ flakes through the phase change and then back to room temperature. 


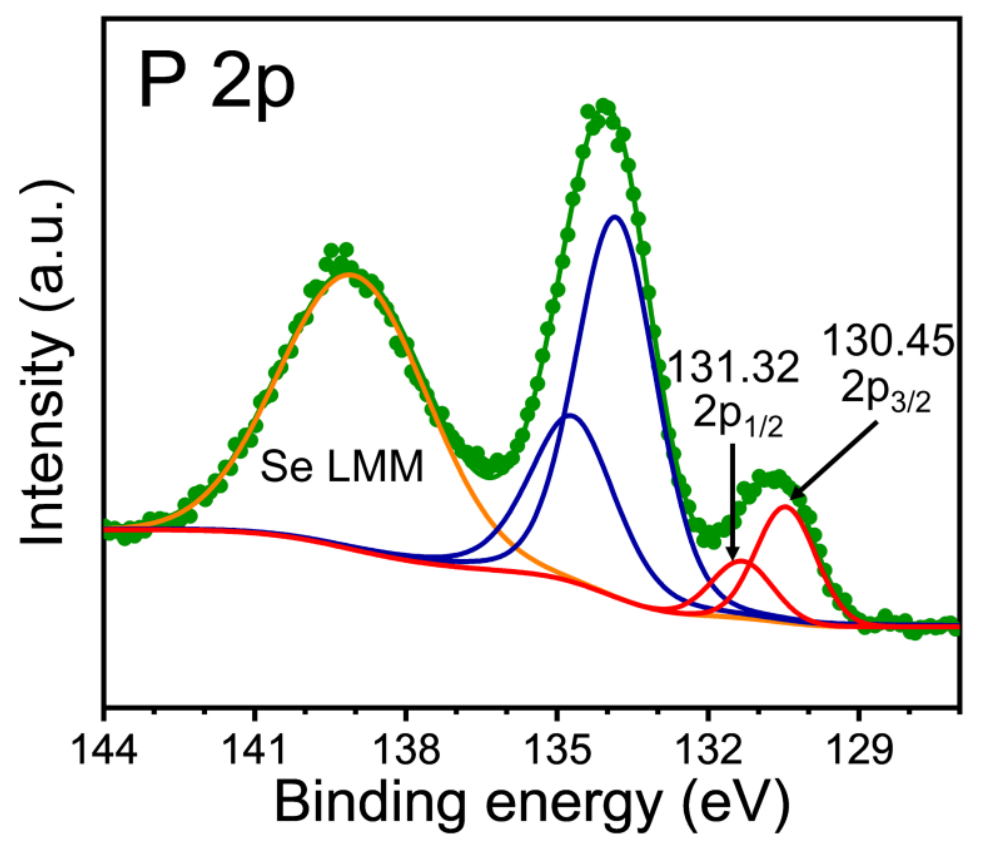

Figure S9. XPS spectrum of $\mathrm{P} 2 p$ for the $\mathrm{P}_{4} \mathrm{Se}_{3}$ nanoflake after plastic-crystal transition. 

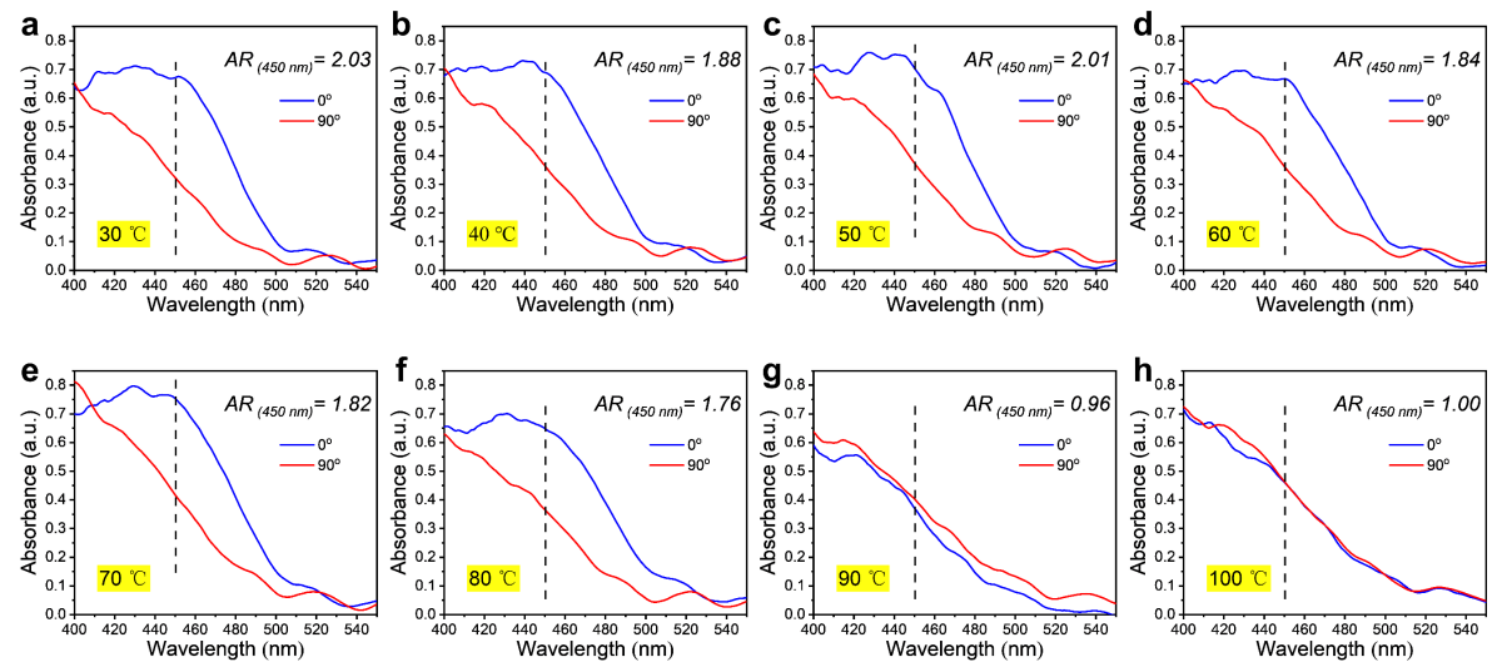

Figure S10. Polarized absorption spectra of a $\mathrm{P}_{4} \mathrm{Se}_{3}$ nanoflake at $0^{\circ}$ and $90^{\circ}$ direction with temperature ranging from $30{ }^{\circ} \mathrm{C}$ to $100{ }^{\circ} \mathrm{C}$ and the corresponding absorbance ratio (AR) at $450 \mathrm{~nm}$. 

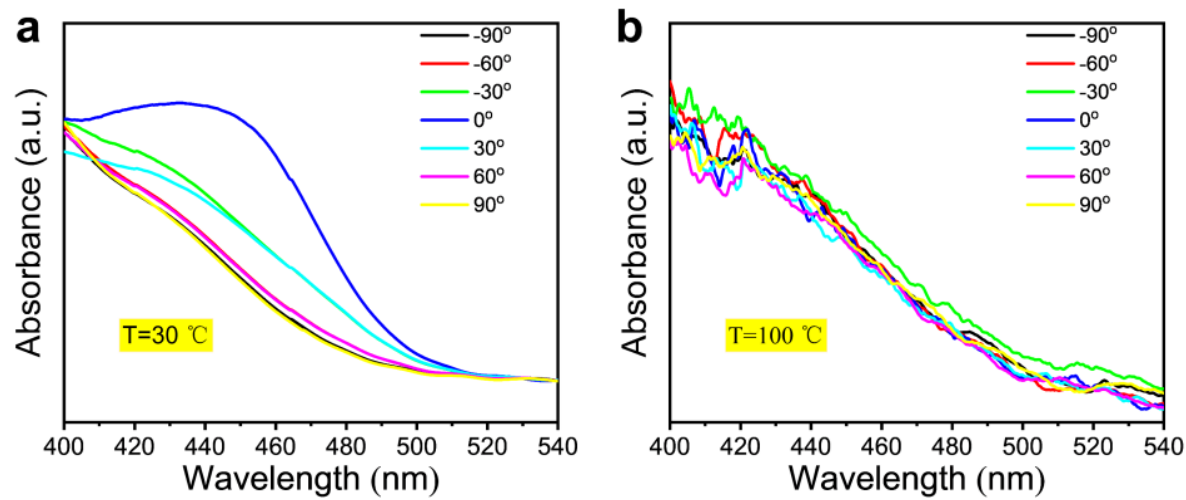

Figure S11. Polarization-resolved absorption spectra of the $\mathrm{P}_{4} \mathrm{Se}_{3}$ nanoflake at $30{ }^{\circ} \mathrm{C}$ and $100{ }^{\circ} \mathrm{C}$, respectively. 
Table S1 Crystallographic data, data collection and structure refinement parameters of $2 \mathrm{D} \alpha-\mathrm{P}_{4} \mathrm{Se}_{3}$ nanoflakes.

\begin{tabular}{|c|c|}
\hline \multicolumn{2}{|c|}{ Crystal data } \\
\hline Chemical formula & $\mathrm{P}_{4} \mathrm{Se}_{3}$ \\
\hline$M_{\mathrm{r}}$ & 360.78 \\
\hline Crystal system, space group & Orthorhombic, Pnma \\
\hline Temperature $(\mathrm{K})$ & 288 \\
\hline$a, b, c(\AA)$ & $11.8044(5), 9.7525(4), 26.2735(10)$ \\
\hline$V\left(\AA^{3}\right)$ & $3024.7(2)$ \\
\hline$Z$ & 16 \\
\hline \multicolumn{2}{|c|}{ Data collection } \\
\hline Diffractometer & $\mathrm{CCD}$ area detector \\
\hline Absorption correction & Multi-scan \\
\hline$T_{\min }, T_{\max }$ & $0.392,0.703$ \\
\hline $\begin{array}{l}\text { No. of measured, independent and observed } \\
{[I>2 \sigma(I)] \text { reflections }}\end{array}$ & $46809,2834,1960$ \\
\hline$R_{\text {int }}$ & 0.167 \\
\hline$(\sin \theta / \lambda)_{\max }\left(\AA^{-1}\right)$ & 0.595 \\
\hline \multicolumn{2}{|c|}{ Refinement } \\
\hline$R\left[F^{2}>2 \sigma\left(F^{2}\right)\right], w R\left(F^{2}\right), S$ & $0.046,0.116,1.04$ \\
\hline No. of reflections & 2834 \\
\hline No. of parameters & 145 \\
\hline$\Delta \rho_{\max }, \Delta \rho_{\min }\left(\mathrm{e} \AA^{-3}\right)$ & $1.51,-0.99$ \\
\hline
\end{tabular}


Table S2 XPS data of the as-synthesized 2D $\mathrm{P}_{4} \mathrm{Se}_{3}$ nanoflakes.

\begin{tabular}{ccccc}
\hline P & $\mathbf{2} \mathbf{p}_{\mathbf{1} / \mathbf{2}}$ & \multicolumn{2}{c}{$\mathbf{2} \mathbf{p}_{\mathbf{3} / \mathbf{2}}$} \\
BE/eV & 131.71 & \multicolumn{2}{c}{130.84} & \\
area & \multicolumn{2}{c}{3057.23} & \multicolumn{2}{c}{ Noncoupled Se } \\
\hline \multirow{2}{*}{ Se } & \multicolumn{2}{c}{ Coupled Se } & $3 \mathrm{~d}_{3 / 2}$ & $3 \mathrm{~d}_{5 / 2}$ \\
& $3 \mathrm{~d}_{3 / 2}$ & $3 \mathrm{~d}_{5 / 2}$ & 56.03 & 55.23 \\
BE/eV & 57.10 & 56.30 & 2152.88 & 3229.33 \\
area & 4083.78 & 6125.67 & & \\
\hline
\end{tabular}


Table S3 Comparison of the temperature dependence coefficients of our $\mathrm{P}_{4} \mathrm{Se}_{3}$ nanoflakes and other van der Waals solids.

\begin{tabular}{ccc}
\hline Materials & $\chi\left(\times \mathbf{1 0}^{-\mathbf{2}} \mathbf{c m}^{-\mathbf{1}} \mathbf{K}^{-\mathbf{1}}\right)$ & References \\
\hline $\mathrm{MoS}_{2}$ & -1.32 & 13 \\
$\mathrm{WS}_{2}$ & -1.28 & 14 \\
$\mathrm{MoSe}_{2}$ & -0.96 & 15 \\
$\mathrm{RhI}_{3}$ & -0.793 & 16 \\
$\mathrm{MnPSe}_{3}$ & -1.01 & 17 \\
$\beta-\mathrm{HgI}_{2}\left(\mathrm{Mol}_{-}-\mathrm{Cry}\right)$ & -0.96 & 18 \\
$\alpha-\mathrm{P}_{4} \mathrm{Se}_{3}$ & -1.41 & This work \\
\hline
\end{tabular}


Table S4 The inter-molecular transfer integral for $\mathrm{P}_{4} \mathrm{Se}_{3}$ nanoflakes calculated at B3LYP/6-311+G** level based on single crystal stacking.

\begin{tabular}{|c|c|c|c|c|c|c|c|}
\hline Molecule 1 & Molecule 2 & $\begin{array}{l}\text { V(hole) } \\
(\mathrm{meV})\end{array}$ & $\begin{array}{c}\mathrm{V} \text { (electron) } \\
(\mathrm{meV})\end{array}$ & Molecule 1 & Molecule 2 & $\begin{array}{c}\text { V(hole) } \\
\text { (meV) }\end{array}$ & $\begin{array}{c}\mathrm{V} \text { (electron) } \\
(\mathrm{meV})\end{array}$ \\
\hline 1 & 12 & -77.979 & 48.931 & 9 & 14 & -77.979 & 48.931 \\
\hline 1 & 9 & 35.442 & -84.117 & 9 & 1 & 35.442 & -84.117 \\
\hline 1 & 15 & -72.236 & -35.767 & 9 & 13 & -72.236 & -35.767 \\
\hline 1 & 14 & -34.375 & -11.866 & 9 & 12 & -34.375 & -11.866 \\
\hline 1 & 8 & 0.173 & -11.228 & 9 & 10 & 0.173 & -11.228 \\
\hline 1 & 3 & 50.923 & -14.144 & 9 & 7 & 50.923 & -14.144 \\
\hline 1 & 10 & 0.047 & 6.93 & 9 & 8 & 0.047 & 6.93 \\
\hline 2 & 7 & -0.05 & 47.141 & 10 & 6 & -185.541 & -40.901 \\
\hline 2 & 8 & -185.541 & -40.901 & 10 & 3 & 0.043 & -56.539 \\
\hline 2 & 11 & -77.979 & 48.931 & 10 & 16 & 64.701 & 23.869 \\
\hline 2 & 4 & -15.241 & -41.168 & 10 & 12 & -15.241 & -41.168 \\
\hline 2 & 5 & -34.375 & -11.866 & 10 & 9 & 0.173 & -11.228 \\
\hline 2 & 3 & 0.029 & -112.132 & 10 & 15 & 45.5 & -47.55 \\
\hline 2 & 6 & -24.458 & 33.457 & 10 & 1 & 0.047 & 6.93 \\
\hline 2 & 7 & 0.004 & -34.448 & 11 & 2 & -77.979 & 48.931 \\
\hline 3 & 6 & -0.05 & 47.141 & 11 & 5 & 35.442 & -84.117 \\
\hline 3 & 10 & 0.043 & -56.539 & 11 & 7 & -72.236 & -35.767 \\
\hline 3 & 15 & -21.159 & 54.844 & 11 & 6 & -34.375 & -11.866 \\
\hline 3 & 5 & -72.236 & -35.767 & 11 & 16 & 0.173 & -11.228 \\
\hline 3 & 16 & 45.5 & -47.55 & 11 & 13 & 50.923 & -14.144 \\
\hline 3 & 2 & 0.029 & -112.132 & 11 & 4 & 0.047 & 6.93 \\
\hline 3 & 1 & 50.923 & -14.144 & 12 & 15 & -0.05 & 47.141 \\
\hline 3 & 6 & 0.004 & -34.448 & 12 & 16 & -185.541 & -40.901 \\
\hline 4 & 14 & -185.541 & -40.901 & 12 & 1 & -77.979 & 48.931 \\
\hline 4 & 13 & 0.043 & -56.539 & 12 & 10 & -15.241 & -41.168 \\
\hline 4 & 8 & 64.701 & 23.869 & 12 & 9 & -34.375 & -11.866 \\
\hline 4 & 2 & -15.241 & -41.168 & 12 & 13 & 0.029 & -112.132 \\
\hline 4 & 5 & 0.173 & -11.228 & 12 & 14 & -24.458 & 33.457 \\
\hline 4 & 7 & 45.5 & -47.55 & 12 & 15 & 0.004 & -34.448 \\
\hline 4 & 11 & 0.047 & 6.93 & 13 & 14 & -0.05 & 47.141 \\
\hline 5 & 6 & -77.979 & 48.931 & 13 & 4 & 0.043 & -56.539 \\
\hline 5 & 11 & 35.442 & -84.117 & 13 & 7 & -21.159 & 54.844 \\
\hline 5 & 3 & -72.236 & -35.767 & 13 & 9 & -72.236 & -35.767 \\
\hline
\end{tabular}




\begin{tabular}{|c|c|c|c|c|c|c|c|}
\hline 5 & 2 & -34.375 & -11.866 & 13 & 8 & 45.5 & -47.55 \\
\hline 5 & 4 & 0.173 & -11.228 & 13 & 12 & 0.029 & -112.132 \\
\hline 5 & 15 & 50.923 & -14.144 & 13 & 11 & 50.923 & -14.144 \\
\hline 5 & 16 & 0.047 & 6.93 & 13 & 14 & 0.004 & -34.448 \\
\hline 6 & 3 & -0.05 & 47.141 & 14 & 13 & -0.05 & 47.141 \\
\hline 6 & 10 & -185.541 & -40.901 & 14 & 4 & -185.541 & -40.901 \\
\hline 6 & 5 & -77.979 & 48.931 & 14 & 9 & -77.979 & 48.931 \\
\hline 6 & 16 & -15.241 & -41.168 & 14 & 8 & -15.241 & -41.168 \\
\hline 6 & 11 & -34.375 & -11.866 & 14 & 1 & -34.375 & -11.866 \\
\hline 6 & 7 & 0.029 & -112.132 & 14 & 15 & 0.029 & -112.132 \\
\hline 6 & 2 & -24.458 & 33.457 & 14 & 12 & -24.458 & 33.457 \\
\hline 6 & 3 & 0.004 & -34.448 & 14 & 13 & 0.004 & -34.448 \\
\hline 7 & 2 & -0.05 & 47.141 & 15 & 12 & -0.05 & 47.141 \\
\hline 7 & 8 & 0.043 & -56.539 & 15 & 16 & 0.043 & -56.539 \\
\hline 7 & 13 & -21.159 & 54.844 & 15 & 3 & -21.159 & 54.844 \\
\hline 7 & 11 & -72.236 & -35.767 & 15 & 1 & -72.236 & -35.767 \\
\hline 7 & 4 & 45.5 & -47.55 & 15 & 10 & 45.5 & -47.55 \\
\hline 7 & 6 & 0.029 & -112.132 & 15 & 14 & 0.029 & -112.132 \\
\hline 7 & 9 & 50.923 & -14.144 & 15 & 5 & 50.923 & -14.144 \\
\hline 7 & 2 & 0.004 & -34.448 & 15 & 12 & 0.004 & -34.448 \\
\hline 8 & 2 & -185.541 & -40.901 & 16 & 12 & -185.541 & -40.901 \\
\hline 8 & 7 & 0.043 & -56.539 & 16 & 15 & 0.043 & -56.539 \\
\hline 8 & 4 & 64.701 & 23.869 & 16 & 10 & 64.701 & 23.869 \\
\hline 8 & 14 & -15.241 & -41.168 & 16 & 6 & -15.241 & -41.168 \\
\hline 8 & 1 & 0.173 & -11.228 & 16 & 11 & 0.173 & -11.228 \\
\hline 8 & 13 & 45.5 & -47.55 & 16 & 3 & 45.5 & -47.55 \\
\hline 8 & 9 & 0.047 & 6.93 & 16 & 5 & 0.047 & 6.93 \\
\hline
\end{tabular}


Table S5 The transfer integral for adjacent $\mathrm{P}_{4} \mathrm{Se}_{3}$ molecules in each layer.

\begin{tabular}{|c|c|c|c|}
\hline Molecule 1 & Molecule 2 & $\begin{array}{c}\text { V(hole) } \\
\text { (meV) }\end{array}$ & $\begin{array}{c}\mathrm{V} \text { (electron) } \\
(\mathrm{meV})\end{array}$ \\
\hline 1 & 12 & -77.979 & 48.931 \\
\hline 10 & 12 & -15.241 & -41.168 \\
\hline 10 & 15 & 45.5 & -47.55 \\
\hline 1 & 15 & -72.236 & -35.767 \\
\hline 8 & 13 & 45.5 & -47.55 \\
\hline 9 & 13 & -72.236 & -35.767 \\
\hline 9 & 14 & -77.979 & 48.931 \\
\hline 8 & 14 & -15.241 & -41.168 \\
\hline 2 & 11 & -77.979 & 48.931 \\
\hline 7 & 11 & -72.236 & -35.767 \\
\hline 4 & 7 & 45.5 & -47.55 \\
\hline 4 & 2 & -15.241 & -41.168 \\
\hline 3 & 16 & 45.5 & -47.55 \\
\hline 6 & 16 & -15.241 & -41.168 \\
\hline 5 & 6 & -77.979 & 48.931 \\
\hline 5 & 3 & -72.236 & -35.767 \\
\hline 12 & 15 & -0.05 & 47.141 \\
\hline 1 & 10 & 0.047 & 6.93 \\
\hline 14 & 13 & -0.05 & 47.141 \\
\hline 8 & 9 & 0.047 & 6.93 \\
\hline 2 & 7 & -0.05 & 47.141 \\
\hline 4 & 11 & 0.047 & 6.93 \\
\hline 3 & 6 & -0.05 & 47.141 \\
\hline 5 & 16 & 0.047 & 6.93 \\
\hline
\end{tabular}


Table S6 The transfer integrals of hole for $\mathrm{P}_{4} \mathrm{Se}_{3}$ molecules between adjacent layers.

\begin{tabular}{|c|c|c|}
\hline Molecule 1 & Molecule 2 & $\begin{array}{c}\text { V(hole) } \\
(\mathrm{meV})\end{array}$ \\
\hline 16 & 12 & -185.541 \\
\hline 8 & 2 & -185.541 \\
\hline 6 & 10 & -185.541 \\
\hline 4 & 14 & -185.541 \\
\hline 10 & 16 & 64.701 \\
\hline 4 & 8 & 64.701 \\
\hline 15 & 5 & 50.923 \\
\hline 13 & 11 & 50.923 \\
\hline 7 & 9 & 50.923 \\
\hline 1 & 3 & 50.923 \\
\hline 5 & 11 & 35.442 \\
\hline 1 & 9 & 35.442 \\
\hline 9 & 12 & -34.375 \\
\hline 6 & 11 & -34.375 \\
\hline 2 & 5 & -34.375 \\
\hline 1 & 14 & -34.375 \\
\hline 12 & 14 & -24.458 \\
\hline 6 & 2 & -24.458 \\
\hline 2 & 6 & -24.458 \\
\hline 13 & 7 & -21.159 \\
\hline 3 & 15 & -21.159 \\
\hline 11 & 16 & 0.173 \\
\hline 9 & 10 & 0.173 \\
\hline 4 & 5 & 0.173 \\
\hline 1 & 8 & 0.173 \\
\hline 15 & 16 & 0.043 \\
\hline 7 & 8 & 0.043 \\
\hline 4 & 13 & 0.043 \\
\hline 3 & 10 & 0.043 \\
\hline 2 & 3 & 0.029 \\
\hline 15 & 14 & 0.029 \\
\hline 12 & 13 & 0.029 \\
\hline 6 & 7 & 0.029 \\
\hline
\end{tabular}


Table S7 The transfer integrals of electron for $\mathrm{P}_{4} \mathrm{Se}_{3}$ molecules between adjacent layers.

\begin{tabular}{|c|c|c|}
\hline Molecule 1 & Molecule 2 & $\begin{array}{c}\mathrm{V} \text { (electron) } \\
(\mathrm{meV})\end{array}$ \\
\hline 2 & 3 & -112.132 \\
\hline 6 & 7 & -112.132 \\
\hline 12 & 13 & -112.132 \\
\hline 15 & 14 & -112.132 \\
\hline 1 & 9 & -84.117 \\
\hline 5 & 11 & -84.117 \\
\hline 3 & 10 & -56.539 \\
\hline 4 & 13 & -56.539 \\
\hline 7 & 8 & -56.539 \\
\hline 15 & 16 & -56.539 \\
\hline 3 & 15 & 54.844 \\
\hline 13 & 7 & 54.844 \\
\hline 4 & 14 & -40.901 \\
\hline 6 & 10 & -40.901 \\
\hline 8 & 2 & -40.901 \\
\hline 16 & 12 & -40.901 \\
\hline 2 & 6 & 33.457 \\
\hline 6 & 2 & 33.457 \\
\hline 12 & 14 & 33.457 \\
\hline 4 & 8 & 23.869 \\
\hline 10 & 16 & 23.869 \\
\hline 1 & 3 & -14.144 \\
\hline 7 & 9 & -14.144 \\
\hline 13 & 11 & -14.144 \\
\hline 15 & 5 & -14.144 \\
\hline 1 & 14 & -11.866 \\
\hline 2 & 5 & -11.866 \\
\hline 6 & 11 & -11.866 \\
\hline 9 & 12 & -11.866 \\
\hline 1 & 8 & -11.228 \\
\hline 4 & 5 & -11.228 \\
\hline 9 & 10 & -11.228 \\
\hline 11 & 16 & -11.228 \\
\hline
\end{tabular}


Table S8 The calculated reorganization energy for the $16 \mathrm{P}_{4} \mathrm{Se}_{3}$ molecules in unit cell with ONIOM model at B3LYP/6-311+G**:UFF level.

\begin{tabular}{ccc}
\hline \multicolumn{3}{c}{ RE with ONIOM } \\
\hline Molecule 1 & $\begin{array}{c}\text { RE_hole } \\
(\mathrm{eV})\end{array}$ & $\begin{array}{c}\text { RE_electron } \\
(\mathrm{eV})\end{array}$ \\
1 & 0.416 & 0.900 \\
2 & 0.739 & 0.886 \\
3 & 0.379 & 0.876 \\
4 & 0.378 & 1.247 \\
5 & 0.416 & 0.900 \\
6 & 0.735 & 0.886 \\
7 & 0.379 & 0.876 \\
8 & 0.376 & 1.247 \\
9 & 0.416 & 0.900 \\
10 & 0.374 & 1.247 \\
11 & 0.416 & 0.900 \\
12 & 0.739 & 0.886 \\
13 & 0.378 & 0.876 \\
14 & 0.739 & 0.886 \\
15 & 0.379 & 0.876 \\
16 & 0.377 & 1.247 \\
\hline & &
\end{tabular}


Table S9 Calculated hole and electron mobility of $\alpha-\mathrm{P}_{4} \mathrm{Se}_{3}$ crystal by Marcus theory and quantum nuclear tunneling model.

\begin{tabular}{ccc}
\hline Mobility & $\begin{array}{c}\text { Hole } \\
\left(\times \mathbf{1 0}^{-1} \mathbf{c m}^{\mathbf{2}} \mathbf{V}^{-\mathbf{1}} \mathbf{s}^{-\mathbf{1}}\right)\end{array}$ & $\begin{array}{c}\text { Electron } \\
\left(\times \mathbf{1 0} \mathbf{1}^{-\mathbf{3}} \mathbf{c m}^{\mathbf{2}} \mathbf{V}^{\mathbf{- 1}} \mathbf{s}^{\mathbf{- 1}}\right)\end{array}$ \\
\hline Mob_a_marcus & 0.49 & 1.01 \\
Mob_b_marcus & 2.91 & 0.35 \\
Mob_c_marcus & 0.08 & 0.17 \\
Mob_ave_marcus & 1.16 & 0.51 \\
\hline Mob_a_quantum & 0.72 & 2.24 \\
Mob_b_quantum & 4.01 & 0.77 \\
Mob_c_quantum & 0.11 & 0.35 \\
Mob_ave_quantum & 1.62 & 1.12 \\
\hline
\end{tabular}




\section{References}

(1) Sheldrick, G. M., Crystal structure refinement with SHELXL. Acta Crystallogr. C Struct. Chem. 2015, 71, 3-8.

(2) Kresse, G.; Hafner, J., Ab initio molecular dynamics for liquid metals. Phys. Rev. $B$ 1993, 47, 558-561.

(3) Perdew, J. P.; Burke, K.; Ernzerhof, M., Generalized Gradient Approximation Made Simple. Phys. Rev. Lett. 1996, 77, 3865-3868.

(4) Marcus, R. A., Electron transfer reactions in chemistry. Theory and experiment. Rev. Mod. Phys. 1993, 65, 599-610.

(5) Nan, G.; Yang, X.; Wang, L.; Shuai, Z.; Zhao, Y., Nuclear tunneling effects of charge transport in rubrene, tetracene, and pentacene. Phys. Rev. B 2009, 79, 115203.

(6) Shuai, Z.; Geng, H.; Xu, W.; Liao, Y.; Andre, J. M., From charge transport parameters to charge mobility in organic semiconductors through multiscale simulation. Chem. Soc. Rev. 2014, 43, 2662-2679.

(7) Wang, L.; Nan, G.; Yang, X.; Peng, Q.; Li, Q.; Shuai, Z., Computational methods for design of organic materials with high charge mobility. Chem. Soc. Rev. 2010, 39, 423-434.

(8) Shuai, Z.; Li, W.; Ren, J.; Jiang, Y.; Geng, H., Applying Marcus theory to describe the carrier transports in organic semiconductors: Limitations and beyond. J. Chem. Phys. 2020, 153, 080902.

(9) Peng, X.; Li, Q.; Shuai, Z., Influences of dynamic and static disorder on the carrier mobility of BTBT-C12 derivatives: a multiscale computational study. Nanoscale 2021, $13,3252-3262$.

(10) Niu, Y.; Li, W.; Peng, Q.; Geng, H.; Yi, Y.; Wang, L.; Nan, G.; Wang, D.; Shuai, Z., MOlecular MAterials Property Prediction Package (MOMAP) 1.0: a software package for predicting the luminescent properties and mobility of organic functional materials. Mol. Phys. 2018, 116, 1078-1090.

(11) Aubauer, C.; Irran, E.; Klapotke, T. M.; Schnick, W.; Schulz, A.; Senker, J., A theoretical and experimental study on the Lewis acid-base adducts $\left(\mathrm{P}_{4} \mathrm{E}_{3}\right) \cdot\left(\mathrm{BX}_{3}\right)(\mathrm{E}=$ 
$\mathrm{S}, \mathrm{Se} ; \mathrm{X}=\mathrm{Br}, \mathrm{I})$ and $\left(\mathrm{P}_{4} \mathrm{Se}_{3}\right) \cdot\left(\mathrm{NbCl}_{5}\right)$. Inorg. Chem. 2001, 40, 4956-4965.

(12) Fratini, S.; Ciuchi, S.; Mayou, D.; de Laissardiere, G. T.; Troisi, A., A map of high-mobility molecular semiconductors. Nat. Mater. 2017, 16, 998-1002.

(13) Sahoo, S.; Gaur, A. P. S.; Ahmadi, M.; Guinel, M. J. F.; Katiyar, R. S., Temperature-Dependent Raman Studies and Thermal Conductivity of Few-Layer $\mathrm{MoS}_{2}$.J. Phys. Chem. C 2013, 117, 9042-9047.

(14) Peimyoo, N.; Shang, J.; Yang, W.; Wang, Y.; Cong, C.; Yu, T., Thermal conductivity determination of suspended mono- and bilayer $\mathrm{WS}_{2}$ by Raman spectroscopy. Nano Res. 2014, 8, 1210-1221.

(15) Pawbake, A. S.; Pawar, M. S.; Jadkar, S. R.; Late, D. J., Large area chemical vapor deposition of monolayer transition metal dichalcogenides and their temperature dependent Raman spectroscopy studies. Nanoscale 2016, 8, 3008-3018.

(16) Wang, F.; Zhang, Z.; Zhang, Y.; Nie, A.; Zhao, W.; Wang, D.; Huang, F.; Zhai, T., Honeycomb $\mathrm{RhI}_{3}$ Flakes with High Environmental Stability for Optoelectronics. $A d v$. Mater. 2020, 32, 2001979.

(17) Liu, G.; Su, J.; Feng, X.; Li, H.; Zhai, T., Synthesis of 2D ternary layered manganese phosphorous trichalcogenides towards ultraviolet photodetection. Sci. China Mater. 2021, 64, 2251-2260.

(18) Lin, Z.; Ding, Y.; Zheng, W.; Zhu, Y.; Zhu, S.; Huang, F., 2D van der Waals Molecular Crystal $\beta-\mathrm{HgI}_{2}$ : Economical, Rapid, and Substrate-Free Liquid-Phase Synthesis and Strong In-Plane Optical Anisotropy. Small 2021, 17, 2005368. 